\title{
Nutritional State Impact on the Liver Detoxification Function in Patients Infected by HIV under Antiretroviral Drugs at the Bertoua Day Hospital of Cameroon
}

\author{
Marcellin Guiaro Ndoe ${ }^{1}$, Armel Herve Nwabo Kamdje ${ }^{1}$, Charles Fokunang Ntungwen², \\ Alexandre Michel Njan Nloga ${ }^{3}$ \\ ${ }^{1}$ Department of Biomedical Sciences, Faculty of Science, University of Ngaoundere, Ngaoundere, Cameroon \\ ${ }^{2}$ Faculty of Medicine and Biomedical Sciences, University of Yaounde I, Yaounde, Cameroon \\ ${ }^{3}$ Department of Biological Sciences, Faculty of Science, University of Ngaoundere, Ngaoundere, Cameroon
}

Email address:

d.nokosore@yahoo.fr (M. G. Ndoe)

\section{To cite this article:}

Marcellin Guiaro Ndoe, Armel Herve Nwabo Kamdje, Charles Fokunang Ntungwen, Alexandre Michel Njan Nloga. Nutritional State Impact on the Liver Detoxification Function in Patients Infected by HIV under Antiretroviral Drugs at the Bertoua Day Hospital of Cameroon.

Journal of Diseases and Medicinal Plants. Vol. 1, No. 2, 2015, pp. 37-41. doi: 10.11648/j.jdmp.20150102.13

\begin{abstract}
The present study aimed at evaluating the nutritional state impact on liver detoxification function in patients infected by the human immunodeficiency virus under antiretroviral drugs. We performed a retrospective and prospective transversal survey on the patients infected by the human immunodeficiency virus, under antiretroviral drugs at the Bertoua day hospital. The retrospective period of our survey went from October 24, 2014 to February 24,2014 (4 months) and the prospective shutter of the survey went from February 24, 2014 to August 24, 2014 (6 months), a total period of 10 months. We also analysed the socio-demographics parameters (Age and Sex), Biological parameters (HIV type, CD4, Transaminases) and the therapeutics parameters (Therapeutic protocol, Evolution). The hepatic affection has been judged by the remarkable increase of the rate of transaminases $(>3 \mathrm{~N})$ and by the appearance of hypersensitivity signs (Skin rashes, Fever...). During this survey, 38 hepatic-toxicity cases caused by the intake of ARV drugs were collected. We included 12 men (31.58\%) and 26 women $(68.42 \%)$ with a sex-ratio of 0.46 in favor of the women. The average age was $45.68 \pm 13.13$ year with age ranging between 10 to 65 years. Age group of 50 years and above was most represented with a percentage of 42.10 . The majority of the patients were infected by the HIV $1(86.85 \%)$ against $10.52 \%$ for the HIV 2 and $2.63 \%$ for the HIV $1+2$. The most prescribed therapy was the association of 2 INTI+1 INNTI (Tenofovir+Lamivudine+Nevirapine) and the AIDS stage represented $7.89 \%$. Prior to the antiretroviral therapy, the 38 patients had normal transaminases that followed modifications of the initial therapeutic protocol after a remarkable increase during the antiretroviral therapy. We noted during this survey that the hepatotoxicity intervention of our patients under ARV drugs was correlated to their Body Mass Index (BMI) that allowed us to evaluate the nutritional state of our patients. The intervention of the hepatotoxicity during the antiretroviral therapy, among the HIV patients having a good nutritional state (normal BMI), was very weak in the absence of the predisposing factors.
\end{abstract}

Keywords: Hepatic-toxicity, Transaminase, ARV, HIV, BMI

\section{Introduction}

AIDS has been for more than 30 years [1].The first cases were described in the United States of America in 1981. The pathogenic agent that is the human immunodeficiency virus (HIV) was discovered in France in 1983. The first antiretroviral drugs, that is zidovudine, was used in 1986 and the efficient therapeutic combinations in 1996 [1]. According to the report of UNO/AIDS 2012, the evolution of the AIDS/HIV epidemic in the world has taken a new face during these last years [1]. The number of People Living with the HIV (PLHIV) at the end of 2011 was estimated at 34.2 million, a rise of $17 \%$ compared to 2001 . This rise is due to a significant expansion of the access to the antiretroviral therapy that contributes to the reduction of the deaths related to AIDS [1]. In our country Cameroon, the prevalence rate of HIV/AIDS is $4.3 \%$ with about $5.6 \%$ of women carrying this HIV/AIDS as against $2.9 \%$ for men [1]. In the East region of Cameroon, where our survey took place, the prevalence rate was $6.3 \%$ with $8.8 \%$ of carrier women of the AIDS/HIV 
against $3.7 \%$ of carrier men [2]. The antiretroviral therapy comes to transform the natural story of the HIV infection, with a meaningful reduction notably linked to the morbidity and mortality caused by opportunist infections. Also this therapy overflows many undesirable effects complicating the handling of the HIV infection. Hepatic-toxicity holds among these undesirable effects, an important place in the management of patient therapy. It has as consequence, to be responsible for an increase of therapy stoppage about $6 \%$ in 1996 to about 32\% between 1998 and 1999 [3]. These therapy stoppages could have a deleterious effect on the handling of the patients and the impact of a severe hepatictoxicity under antiretroviral therapy which varies from 2 to $18 \%$ [3]. Most of the studies on the hepatic-toxicity of ARV drugs have been carried out in Europe, Asia, America and very little in Africa. These studies were carried out on patients predisposed to the hepatic-toxicity factors of antiretroviral therapy for example the viral coinfection (HIV/HCV or HVB). In Cameroon to the best of our knowledge, no survey has been carried out on patients infected with the human immunodeficiency virus with the absence of these predisposing factors. It is for this reason that we judged its necessary and appropriate to conduct this survey to determine the intervening reason of this undesirable effect among the HIV patients, under ARV drugs, either even possible, in spite of the absence of these predisposing factors. To arrive at this aim, we threw our attention on the nutritional state of these patients under ARV drugs, in order to value its impact on the detoxification function of the liver after having evaluated the frequency of the hepatic-toxicity cases linked to the intake of ARV drugs at the Bertoua day hospital.

\section{Material and Methods}

We carried out a retrospective and prospective transversal survey on patients infected with the human immunodeficiency virus, place under antiretroviral drugs, followed at the Bertoua day hospital. The retrospective period of our survey went from October 24, 2014 to February 24, 2014 (4 months) and the prospective shutter of the survey went from February 24, 2014 to August 24, 2014 (6 months), either a total period of 10 months. We included in our survey, all serologically positive HIV patients placed under ARV drugs, registered at the Bertoua day hospital for at least four months, and presenting a hepatic affection after the intake of ARV drugs with absence of other causes of hepatic attack. We excluded in our survey, all serologically positive HIV patients, unrecorded at the Bertoua day hospital for the follow-up of the antiretroviral therapy; all serologically Positive HIV patients having a bad antiretroviral therapy observance; all serologically positive HIV patients presenting an increase of transaminases occurring out of the antiretroviral therapy. The data analysis was also about the socio-demographic parameters (Age and Sex), Biological parameters (HIV type, CD4, Transaminase) and therapeutic parameters (therapeutic protocol, Evolution). The hepatic affection has been judged by the remarkable increase of the rate of transaminases $(>3 \mathrm{~N})$ and by the appearance of hypersensitivity signs (skin rashes, fever...). The introverted data were typed and analysed with the help of the software Excel 2010 and R.2.13.0. We used the chi-square test to compare the percentages and the accepted significance was fixed to $5 \%$.

\section{Results}

Two hundred and fifty six (256) HIV patients were followed up at the Bertoua day hospital during this period of survey. 176 patients had a good antiretroviral therapy observance. Among these 176 patients, 38 hepatic- toxicity cases caused by the intake of ARV drugs were counted, representing on a frequency of $21.6 \%$.

\subsection{Variation of the Hepatic-toxicity of Antiretroviral Therapy According to the Age Groups}

The mean age was $45.68 \pm 13.13$ year, with ages ranging between 10 to 65 years. The age groups of 50 years and above consisted of 40 to 49 years were the most represented with respective percentages of $42.10 \%$ and $34.22 \%$. This percentages difference was statistically meaningful, with a P.value $=15.74 \times 10^{-5}$ (graph 1$)$.

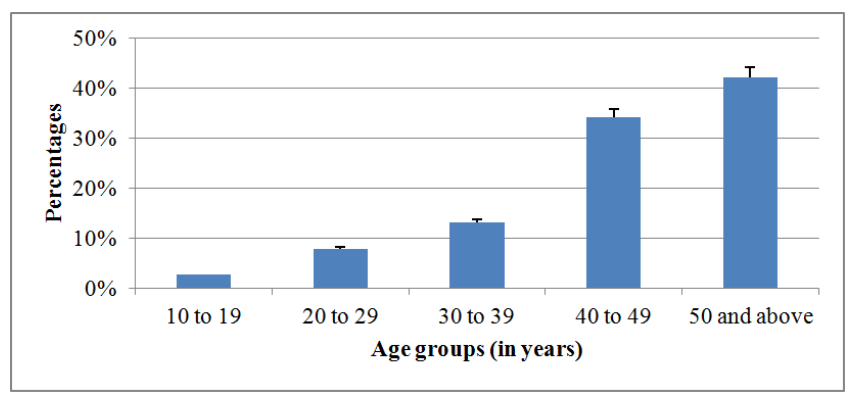

Graph 1. Graphical representation showing the variation of the hepatictoxicity of antiretroviral therapy according to the age groups.

\subsection{Variation of the Hepatic-toxicity of Antiretroviral Therapy According to the Different Sexes}

We counted 12 men and 26 women. The female sex was statistically more predisposed to hepatotoxicity of the antiretroviral therapy, with a Sex-Ratio of 0.46 in favor of women (Graph 2).

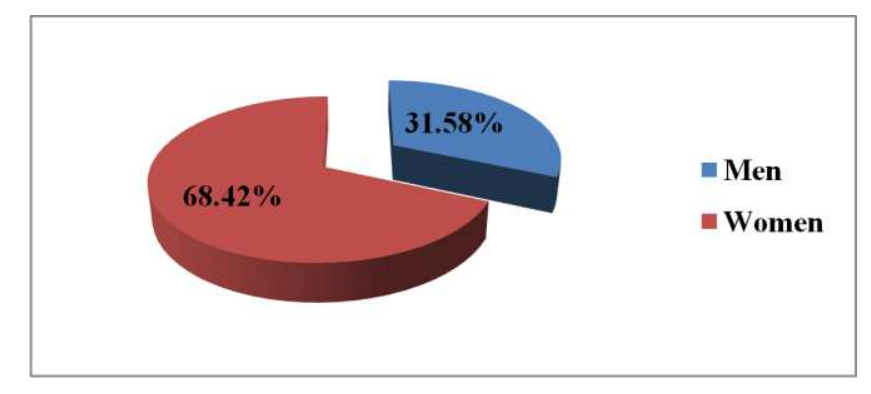

Graph 2. Graphical representation showing variation of the hepatic-toxicity of antiretroviral therapy according to the different sexes. 


\subsection{Initial Therapeutic Protocol}

The most prescribed therapy was the association of 2 INTI+NVP with 23 cases, either a percentages of $60.53 \%$ (Table 1).

Table 1. Showing the patient's distribution according to their initial therapeutic protocol

\begin{tabular}{lll}
\hline Initial Therapeutic Protocol & Numbers & Percentages \\
\hline $\begin{array}{l}\text { 2 INTI + Nevirapine } \\
\text { (INTI = Tenofovir et Lamivudine) }\end{array}$ & 23 & $60.53 \%$ \\
$\begin{array}{l}\text { 2 INTI + Efavirenz } \\
\text { (INTI = Tenofovir ou Zidovudine+ }\end{array}$ & 10 & $26.31 \%$ \\
Lamivudine) & & \\
\hline
\end{tabular}

\begin{tabular}{lll}
\hline Initial Therapeutic Protocol & Numbers & Percentages \\
\hline $\begin{array}{l}\text { Tenofovir + Lamivudine }+ \\
\text { Lopinavir/Ritonavir }\end{array}$ & 5 & $13.16 \%$ \\
Total & 38 & $100 \%$ \\
\hline
\end{tabular}

The differences of percentages were statistically meaningful with a $\mathrm{P}$ value $=0.001$

\subsection{Biological Value of Transaminases Before the Intake of ARV Drugs}

The transaminases (ALAT/ASAT) of 38 patients that we counted were normal before the intake of ARV drugs (Table 2).

Table 2. Showing the patient's distribution of Biological value of transaminases before the intake of ARV drugs

\begin{tabular}{|c|c|c|c|c|}
\hline \multirow{2}{*}{ Transaminases } & \multicolumn{2}{|l|}{ ALAT } & \multicolumn{2}{|l|}{ ASAT } \\
\hline & Numbers & Percentages (\%) & Numbers & Percentages (\%) \\
\hline Normal & 38 & 100 & 38 & 100 \\
\hline Increase & 0 & 0 & 0 & 0 \\
\hline Total & 38 & 100 & 38 & 100 \\
\hline
\end{tabular}

The transaminases of the 38 patients were normal.

\subsection{Modification of the Initial Therapeutic Protocol Following the Increase of Transaminases}

The modifications of the initial therapeutic protocol started from the 2 nd month of the antiretroviral therapy (Table 3).

Table 3. showing the distribution of patients in function of modifications of the initial therapeutic protocol following the increase of transaminases

\begin{tabular}{lllll}
\hline $\begin{array}{l}\text { Initial } \\
\text { Protocol }\end{array}$ & Therapeutic & Numbers & $\begin{array}{l}\text { Percentages } \\
(\%)\end{array}$ & $\begin{array}{l}\text { BMI } \\
(\mathbf{K g} / \mathbf{m} 2)\end{array}$ \\
\hline \multirow{2}{*}{ Modified } & 2 Months & 3 & 7.89 & $>25$ \\
Total & 3 Months & 35 & 92.11 & $<18.5$ \\
\hline
\end{tabular}

The percentage difference was statistically meaningful with a $P$ value $=1.46$ $\mathrm{e}^{-13}$.

\subsection{Normalization of Transaminases and BMI After Modification of the Initial Therapeutic Protocol}

The normalization of transaminase of the 38 patients that knew a modification of the initial therapeutic protocol was correlated to their Body Mass Index (BMI) (Table 4).

Table 4. Showing the distribution of patients on the basis of transaminases normalization and BMI after modification of the initial therapeutic protocol.

\begin{tabular}{lllll}
\hline $\begin{array}{l}\text { Transaminases } \\
\text { Normalization }\end{array}$ & Numbers & $\begin{array}{l}\text { Percentages } \\
(\%)\end{array}$ & BMI \\
\hline No normalized & 29 & 76.31 & Abnormal \\
Normalized & 3 months & 2 & 5.27 & Normal \\
Total & 6 months & 7 & 18.42 & Normal \\
\hline
\end{tabular}

The percentage difference here was not statistically meaningful with a P.value $=0.09$

\subsection{Therapy Evolution}

Most patients that we counted had a steady health state, given 26 patients, having a percentage of $68.43 \%$ (Table 5).
Table 5. Showing the patient's distribution according to their therapy evolution.

\begin{tabular}{lll}
\hline Evolution & Numbers & Percentages (\%) \\
\hline Lost of view & 3 & 7.89 \\
Improved & 6 & 15.79 \\
Stationnary & 26 & 68.43 \\
Died & 3 & 7.89 \\
Total & 38 & 100 \\
\hline
\end{tabular}

The percentage difference was statistically significant with a P.value $=1.87 \mathrm{e}^{-}$ 08

\section{Discussion}

Our survey reported 38 hepatic toxicity cases caused by ARV drugs within a period of 2 years, this was because many cases were not kept in our survey due to the difficulties in regular follow-up of patients (lost of view, transfer, ARV drugs abandoning, death); and a doubt on the intake of drugs or the impossibility to identify the drugs among other drugs taken by the patient placed under ARV drugs. Nevertheless all our hepatic-toxicity cases caused by the intake of ARV drugs have been kept on the basis of a rigorous recruitment supporter counts imputability criteria, notably the absence of other hepatic affections. These different elements have been noted also by many authors. The survey of Lemoine and collaborators in France, concerning an identical population retained 59 cases within 3 years [4], the one of Ponge and collaborators in France retained 5 cases within 6 months [5]. The survey made by Seo and collaborators in Corea, also retained a weak size of sample of 48 cases within 6 years [6]. All these results justifications given out by these studies joined the weak impact of the hepatic-toxicity of drugs in the course of this therapy, consisted between $1 / 10000$ and $1 / 100000$.

The age group of 50 years and above, followed by the age group of 40 to 49 years were the most represented with 
respective rates of $42.10 \%$ and $34.22 \%$. The average age was $45.68 \pm 13.13$ years with age ranging between 10 to 65 years. This result can be justified by the fact that, the antioxidant production by the body, deteriorated with age, more the patient is placed under ARV drugs, more the neutralization capacity of free radicals by this body decreases and therefore predisposes it to a hepatic-toxicity drifting to therapy that the patient takes. The survey of Tanon and collaborators in Côte d'Ivoire, on the efficiency and tolerance of the antiretroviral therapy among aged subjects [7], returned an average age of 60 years. The result justification of this survey was directed at the same sense as ours and stipulated that ageing is a physiological process which entails morphological and functional modifications of the whole body, notably the progressive decrease of the functional capacities of different organs, as well as the decrease of adaptation capacities of the body to different aggressions.

The sex ratio was 0.46 in favor of women. This result justifies itself here by the fact that the follow-up of the antiretroviral therapy is more regular in women than in men, because of their natural physiology (pregnancy/hormonal contraception). In fact there are drugs interactions between PI, NIRT and the oestroprogestatives pills that can generate toxic metabolites for the liver. The efficiency of these pills decreases in the presence of Ritonavir, Lopinavir and Nevirapine [8]. The studies made by Bersoff, Numez and Galli on women infected with HIV, placed under ARV drugs also say that this predisposition to the hepatic-toxicity of the ARV drugs was more than the masculine sex $[9 ; 10 ; 11]$. Accordind to these researchers, the immune restitution is judged efficiently when the CD4 lymphocytes increase by $150-200 \mathrm{~mm}^{3}$ during the first year, then progresses more because the objective of the antiretroviral therapy is to bring back the CD4 lymphocytes to a level superior to $500 / \mathrm{mm}^{3}$. In the presence of Nevirapine, hepatic toxicity occurs in women at the level of CD4 $>250 / \mathrm{mm}^{3}$ and in men at a level of CD $4>400 / \mathrm{mm}^{3}$.

The most widely prescribed therapy was the association of two nucleotidics inhibitors of reverses transcriptase and one non- nucleotidics inhibitors of reverses transcriptase (2 INTI+1 INNTI). This report was also made by Tanon in his survey on the efficiency and tolerance of the antiretroviral therapy among aged subjects in Abidjan [7]. Prior to the antiretroviral therapy, the 38 patients had normal transaminases that followed modifications of the initial therapeutic protocol after a remarkable increase during the antiretroviral therapy. The most hepatotoxic molecule was Nevirapine (23 cases), followed by Efavirenz (10 cases) and these were responsible for immunoallergics hepatitises which causes the modifications of the prescribed initial therapeutic protocol to our patients. During this survey, we noted that the intervention of this immunoallergic hepatitises among our patients placed under ARV drugs, was correlated to their Body Mass Index (BMI). The immunoallergic hepatitises were more visible among patients having a BMI $>25 \mathrm{Kg} / \mathrm{m}^{2}$, because of the weight gain for these patients, that would be responsible for an increase of transaminases related to a hepatic steatosis correlated to the intake of ARV drugs. Then those whose BMI was lower than $18.5 \mathrm{Kg} / \mathrm{m}^{2}$, because a denutrition in these patients that would decrease the capacity of liver detoxification by a decrease in glutathion. For the studies made by other researchers, the NNIRT have an increased risk of immunoallergic hepatitis at the beginning of therapy. With prolonged therapy, the risk of hepatic-toxicity is higher in case of pregnancy, alcohol consumption, in case of CD4 lymphocytes $>250 / \mathrm{mm}^{3}$ of blood in women and $400 / \mathrm{mm}^{3}$ in men, also in case of hepatitis coinfection viruses $[10 ; 12 ; 13]$.

The transaminases normalization after modification of the initial therapeutic protocol was also correlated to the normal Body Mass Index, reflecting the patient's good nutritional state that would prevent the glutathion level reduction of the liver and would also encourage the setting up of the cytoprotection mechanism developed by hepatic cells against oxydative stress induced by the toxic metabolites. On the other hand we noticed a no normalization of transaminases among most patients, that would be due to the presence of anorexia observed in these patients, that is also responsible for denutrition that decreases the capacity of liver detoxification which also leads to a decrease in glutathione; from where there is a persistence of the hepatic cytolysis and no normalization of transaminases among these patients. Six of the patients that knew transaminases normalization (15.79\%), had an improved health.

\section{Conclusion}

We evaluated the nutritional state of our patients through their Body Mass Indexes (BMI). A BMI lower than 18.5 $\mathrm{Kg} / \mathrm{m}^{2}$ reflected a state of gauntness (denutrition); a BMI between $18.5 \mathrm{Kg} / \mathrm{m}^{2}$ to $25 \mathrm{Kg} / \mathrm{m}^{2}$, translated a normal stoutness (good nutritional state). An IMC superior to 25 $\mathrm{Kg} / \mathrm{m}^{2}$ translated a state of overweight. At the end of this survey, we found a prevalence rate of $14.84 \%$ of hepatic toxicity cases caused by ARV drugs at the Bertoua day hospital. We also noted that this undesirable effect was absent among patients in a good nutritional state (normal BMI).

\section{Authorship Contribution}

All authors contributed to the designing, preparation, editing, and final review of the manuscript.

\section{Acknowledgement}

Authors thank the collaborators of their respective institutions for the comments on the manuscript.

\section{References}

[1] Aubry P., 2013. Le Sida tropical. In Epidémiologie des maladies parasitaires. Tome 3.Opportunistes.Editions Médicales Nationales. 78-89. 
[2] OMS. 2011. Revision des stratégies de dépistage du VIH pour la surveillance. $R E H$. 81, 461-464.

[3] Wit FW, Weverling GJ, Weel J, Jurriaans S, Lange JM. 2002. Incidence of and risk factors for severe hepatotoxicity associated with antiretroviral combination therapy. $J$ Infect Dis. 186: 23-31.

[4] Lemoine A, Ouazir K, Sgro C, Clinard F, Allard C, Guilleminet C. 2002. La fréquence des hépatites médicamenteuses est largement sous-estimée en France. Etude de population dans la Nièvre 1997-2000. Gastroenterol Clin Biol. 26 :399-8320.

[5] Ponge T, Conttin S, Fruneau P, Ponge A., 1990. Hépatites médicamenteuses: situation dans la pathologie iatrogène. Résultat d'une enquête prospective. Med Chir Dig. 19: 105109.

[6] Seo JC, Jeon WJ, Park SS, Kim SH, Lee KM, Chae HB, Park SM, Youn SJ., 2006. Clinical experience of 48 acute toxic hepatitis patients. Korean j hepatol. 12(1): 74-81.

[7] Tanon A, Binan Y, Minta D, Ehui E, Ouattara I, Mossou C, Kouakou, Eholié SP, Aoussi E, Bissagnéné E., 2002. Efficiency and safety of antiretroviral treatment among the elderly in Abidjan. $J$ Infect Dis. 39-40.
[8] Kremer H and Sonnenberg-Schwan U., 2003. Women Living with HIV Does Sex and Gender Matter? A Current Literature Review." Eur J Med Res. 8(1): 8-16.

[9] Bersoff-Matcha SJ, Miller WC, Aberg JA, Van Der Horst C, Hamrick Jr, Powderly WG, Mundy LM., 2001. Sex differences in nevirapine rash. Clin Infect Dis. 32(1): 124-9.

[10] Nunez M, Lana R, Mendoza JL, Martin-Carbonero L, Soriano V., 2001. Risk factors for severe hepatic injury after introduction of highly active antiretroviral therapy. $J$ Acquir Immune Defic Syndr. 27: 426-31.

[11] Galli M, Cozzi-Lepri A, Ridolfo A, Gervasoni C, Ravasio L, Corsico L, Gianelli E, Vaccarezza M, Vullo V, Cargnel A., 2002. Incidence of adipose tissue alterat ions in first-line antiretroviral therapy: theLipoICoNa Study."Arch Intern Med. 162(22): 2621-8.

[12] Sulkowski MS, Thomas DL, Chaisson RE, Moore RD., 2000. Hepatotoxicity associated with antiretroviral therapy in adults infected with human immunodeficiency virus and the role of hepatitis C or B virus infection. JAMA. 283:74-80.

[13] Aceti A, Pasquazzi C, Zechini B, De Bac C., 2002. Hepatotoxicity development during antiretroviral therapy containing protease inhibitors in patients with HIV: the role of hepatitis $\mathrm{B}$ and $\mathrm{C}$ virus infection. $J$ Acquir Immune Defic Syndr. 29: 41-8. 